\title{
Dark Neutrinos
}

\author{
A. Nicolaidis \\ Theoretical Physics Department, University of Thessaloniki, Thessaloniki, Greece \\ Email: nicolaid@auth.gr
}

Received July 13, 2012; revised October 25, 2012; accepted November 8, 2012

\begin{abstract}
Solar, atmospheric and reactor neutrino experiments established that neutrinos are massive. It is quite natural then to consider neutrinos as candidate particles for explaining the dark matter in halos around galaxies. We study the gravitational clustering of these neutrinos within a model of a massive core and a surrounding spherical neutrino halo. The neutrinos form a degenerate Fermi gas and a loaded polytropic equation is established. We solve the equation and we obtain the neutrino density in a galaxy, the size of the galaxy and the galactic rotational curves. The available data favor a neutrino with a mass around $10 \mathrm{eV}$. The consequent cosmological implications are examined.
\end{abstract}

Keywords: Neutrinos; Neutrino Mass; Gravitational Clustering; Dark Matter; Polytrope Equation

Solar, atmospheric, reactor and accelerator neutrinos have provided compelling evidence for the existence of neutrino oscillations, implying non-zero neutrino masses $[1,2]$. Solar neutrino oscillations indicate a mass squared difference $\Delta m_{\mathrm{SOL}}^{2}=6 \times 10^{-5} \mathrm{eV}^{2}$ while atmospheric neutrino oscillations suggest $\Delta m_{A T M}^{2}=2 \times 10^{-3} \mathrm{eV}^{2}$. Further, attributing the LSND anomaly to neutrino oscillations implies the existence of a fourth neutrino, a sterile one, with a mass above $1 \mathrm{eV}$ [3]. Cosmology offers further insights on the neutrino mass scales [4-7]. It is inevitable that neutrinos have participated in gravitational clustering around massive galaxies, constituting part of the dark matter. Our purpose is to investigate the neutrino clustering effect, obtaining information on the cosmological presence of massive neutrinos.

The universe today exhibits structure on many scales. Galaxies range in mass, with most bright galaxies having masses of $10^{10}-10^{12} M_{\odot}$. The sizes and distributions of present-day galaxies reflect the spectrum of initial density fluctuations, seeded by random motion or cosmic strings [8]. We expect that each galaxy attracts the cosmological neutrinos in its neighbourhood, creating a neutrino halo of an average mass $M_{v} \approx 3 \times 10^{10} M_{\odot}$.

Our idealized galaxy consists of a spherical massive core (of a mass $M_{c}=\sigma M_{\odot}$ and a radius $r_{c}$ of few Kpc) surrounded by a spherical neutrino halo. Hydrostatic equilibrium prevails

$$
\frac{1}{r^{2}} \frac{\mathrm{d}}{\mathrm{d} r}\left(\frac{r^{2}}{m N} \frac{\mathrm{d} P}{\mathrm{~d} r}\right)=-4 \pi G m N
$$

where $N(r)$ is the neutrino density, $\mathrm{m}$ is the neutrino mass and $P(r)$ is the pressure of the neutrino gas.
Considering that the neutrinos form a degenerate Fermi gas we obtain a polytrope rquation

$$
P=\left(\frac{3}{4 g \pi}\right)^{2 / 3} \frac{h^{2}}{5 m} N^{5 / 3}
$$

with the degeneracy factor $g=2$ (left-handed neutrinos and right-handed antineutrinos). Equations (1) and (2) lead to

$$
\begin{aligned}
& \frac{1}{r^{2}} \frac{\mathrm{d}}{\mathrm{d} r}\left(r^{2} \frac{1}{N^{1 / 3}} \frac{\mathrm{d} N}{\mathrm{~d} r}\right)=-\gamma N \\
& \gamma=m^{3}\left(3 g^{2}\right)^{1 / 3}(4 \pi)^{5 / 3} G / h^{2}
\end{aligned}
$$

Introducing the (dimensionless) variables $p$ and $x$ through

$$
\begin{aligned}
& N(r)=\frac{g}{6 \pi^{2}}\left(\frac{2 m^{2}}{\hbar^{2}}\right)^{3 / 2}\left(\frac{G \sigma M_{\odot} p}{r}\right)^{3 / 2} \\
& r=q x \\
& q=\left(\frac{3 \pi \hbar^{3}}{4 \sqrt{2} g m^{4} G^{3 / 2} M_{\odot}^{1 / 2} \sigma^{1 / 2}}\right)^{2 / 3}
\end{aligned}
$$

Equation (3) becomes

$$
\frac{\mathrm{d}^{2} p}{\mathrm{~d} x^{2}}=-\frac{p^{3 / 2}}{\sqrt{x}} .
$$

The variable $p$ is related to the gravitational potential $V(r)$ through 


$$
p=\frac{r\left(V_{0}-V\right)}{G M_{c}}
$$

$V_{0}$ is the gravitational potemtial at the outer part of the galaxy, where the density vanishes. Indeed the numerical solution of Equation (8) always provides a finite $x_{0}$, where $p\left(x_{0}\right)=0$. The radius $R$ of the galaxy is then $R=q x_{0}$ (Equation (6)). The total mass of the galaxy $\left(M=M_{c}+M_{v}\right)$ is related to the derivative of $p(x)$ at $x_{0}$ by

$$
-\left(x \frac{\mathrm{d} p}{\mathrm{~d} x}\right)_{x=x_{0}}=\frac{M_{c}+M_{v}}{M_{c}}
$$

At $r=r_{c}$ the boundary condition is

$$
p\left(x=x_{c}\right)=1.0
$$

Equation (8) together with the boundary conditions (10) and (11), represents a loaded polytrope [9-13]. Similar mathematical structures appear in the study of a galactic nucleus, a neutron star, or in the Thomas-Fermi description of an atom.

We solved numerically Equation (8), using the boundary condition of Equation (11) and an arbitrary positive value for the derivative of $p(x)$ at $x=x_{c}$. The numerical solution provides a function $p(x)$ which rises up to a maximum, then decreases until it vanishes at some point $x_{0}\left(p\left(x_{0}\right)=0\right)$. We evaluated also the derivative of $p(x)$ in the neighbourhood of $x_{0}$ and subsequently the left-hand side of Equation (10), thus determining the ratio $M_{v} / M_{c}$. To obtain the desired ratio $M_{v} / M_{c}$, we repeat the numerical evaluation with a different value for the derivative of $p(x)$ at $x_{c}$, until we achieve the predetermined $M_{v} / M_{c}$ ratio. The numerical study revealed the following essential features of the loaded polytrope:

1) The precise value of $x_{0}$ is largely independent of the ratio $M_{v} / M_{c}$. More massive neutrino halos provide larger values for the maximum of $p(x)$, but all neutrino densities vanish in the vicinity of $x_{0}=2.0$. Thus the radius $R$ of the galaxy is set up by the constant $q$ (Equations (6) and (7)).

2) The scale q, Equation (7), is very sensitive to the mass of neutrino $m$. By increasing the mass of the neutrino by a factor 3 , the size of the galaxy is reduced by a factor 19. The overall data are well reproduced with a neutrino mass at $10 \mathrm{eV}$.

3) Equation (7) gives then the numerical expression

$$
q=\frac{1.7}{\sigma^{1 / 3}} 10^{5} \mathrm{Kpc}
$$

For a massive core $M_{c}=10^{11} M_{\odot}$, we obtain $q=36 \mathrm{Kpc}$ and therefore $R \approx 70 \mathrm{Kpc}$, in agreement with the data for the size of massive galaxies [14]. It is impressive that a scale $q$ which is expressed in terms of fundamental constants, such as the Planck constant, the neutrino mass and the Newton constant, reproduces accurately the galactic sizes.

4) Through Equation (5) we obtain back the neutrino density $N(r)$. We observe that at small distances, $N(r)$ behaves as

$$
N(r) \sim \frac{1}{r^{3 / 2}} \text { at small } r
$$

while the neutrino density diverges as $r \rightarrow 0$, the total neutrino mass $M_{v}$ remains finite.

Figure 1 shows the neutrino density as a function of the rescaled distance $x$. The upper curve corresponds to $M_{c}=10^{11} M_{\odot}, M_{v}=10 M_{c}$, while the lower curve corresponds to $M_{c}=10^{11} M_{\odot}, M_{v}=M_{c}$. Near the galactic core, the neutrino density is high as $10^{7}$ neutrinos $/ \mathrm{cm}^{3}$, and over the uniform density of the big-bang cosmology, the gravitational clustering provides locally an increase by a factor $10^{5}$.

The spherical neutrino halo up to a rescaled distance $x$, gives a mass $\mu_{v}(x)$, where

$$
\mu_{v}(x)=\sigma M_{\odot} \int y^{1 / 2}[p(y)]^{3 / 2} \mathrm{~d} y .
$$

Obviously $M_{v}=\mu_{v}\left(x_{0}\right)$. The galactic rotational velocity, due to the neutrino halo, is then

$$
u=\left(\frac{G \mu_{v}(r)}{r}\right)^{1 / 2} \text {. }
$$

Figure 2 shows the galactic rotation curves (upper

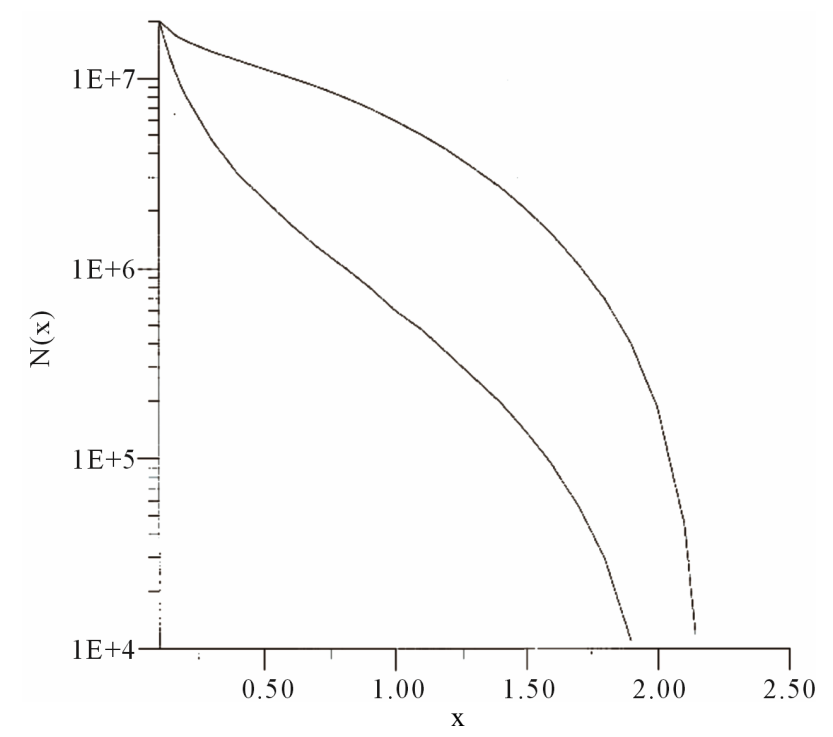

Figure 1. The neutrino density in a spherical galaxy as a function of the rescaled distance $x$. Upper curve corresponds to $M_{v}=M_{c}$, the lower curve corresponds to $M_{v}=M_{c}$. For a $M_{c}=10^{11} M_{\Theta}$, real distance is obtained by multiplying the dimensionless $x$ by 36 Kpc. 


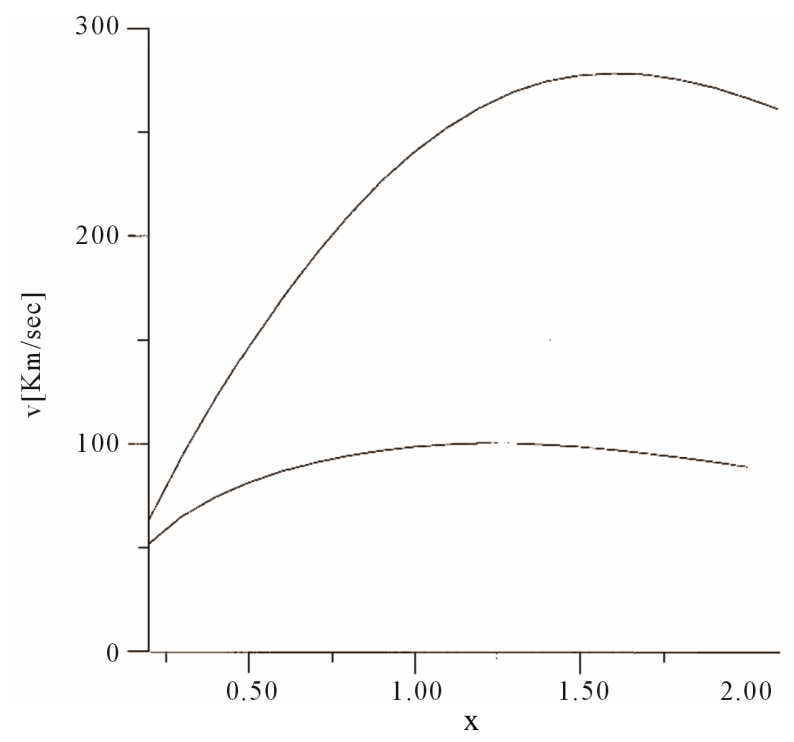

Figure 2. The galactic rotational velocity, due to a spherical neutrino halo, as a function of the rescaled distance $x$. Upper curve corresponds to $M_{v}=M_{c}$, lower to $M_{v}=M_{c}$. For a $M_{c}=10^{11} M_{\odot}$, real distance is obtained by multiplying the dimensionless $x$ by 36 Kpc.

curve corresponds to $M_{c}=10^{11} M_{\odot}, M_{v}=10 M_{c}$, lower curve corresponds to $M_{c}=10^{11} M_{\odot}, M_{v}=M_{c}$ ) and there is agreement with the trends of the experimental data.

We may reach our findings by making appeal to an even simpler model $[15,16]$. A gravitational potential well of the size of the galactic halo is filled up with neutrinos, which are treated like a Fermi-Dirac gas at zero temperature. The total number of neutrinos is related to the Fermi momentum, itself determined by the minimum kinetic energy required for the neutrino to escape from the galaxy. It is found then that the radius of the galaxy is $R=q 3^{2 / 3}$, in full agreement with our results. In another study, the analysis of the neutrino clustering by employing techniques of statistical mechanics favors again a neutrino mass of few $\mathrm{eV}$ [17].

Is it possible to detect the cosmological relic neutrinos? The gravitational clustering effect we studied here, enhances the local densities, however the average neutrino energy is very small and the corresponding weak crosssection (of the order of $10^{-53} \mathrm{~cm}^{2}$ ) renders detection by conventional means highly unlikely [18].

The few $\mathrm{eV}$ mass scale for the sterile neutrino, that our study suggests, fits nicely with the findings of the reactor and short-based neutrino oscillation experiments [3]. On the other hand the cosmological verdict for a few eVmass sterile neutrino is rather unclear. The standard $\Lambda C D M$ framework provides the constraint $m \leq 0.5 \mathrm{eV}$. A modified though $\Lambda C D M$ cosmology, with additional radiation, can accommodate a few eV neutrino [19]. In another direction, there is strong evidence for the exis- tence of a $17 \mathrm{keV}$ massive neutrino [20,21]. The coexistence of distinct mass scales for the neutrinos, sub-eV, $\mathrm{eV}$ and $\mathrm{keV}$, indicates the complexity of neutrino physics. Clearly we need further experimental information and novel theoretical insights to decode the hidden dynamics.

In summary we presented a simplified model for the gravitational clustering of massive neutrinos in the galaxies. There is no adjustable parameter in our analysis and despite its simplicity the overall features of galactic dynamics are reproduced.

\section{Acknowledgements}

I would like to thank Nicos Vlachos for helping me to use the program MATHEMATICA in the numerical work. This research was supported in part by the Templeton Foundation and the EU program "Human Capital and Mobility".

\section{REFERENCES}

[1] A. Romanino, "Neutrino Physics," CERN Yellow Report CERN-2012-001, pp. 153-182.

[2] A. Strumia and F. Vissani, "Neutrino Masses and Mixings and...," arXiv:hep-ph/0606054.

[3] C. Giunti, "Phenomenology of Sterile Neutrinos," arXiv: 1110.3914 [hep-ph].

[4] N. Mavromatos, "Neutrinos and the Universe," Invited Paper to NUFACT11, arXiv:1110.3729 [hep-ph].

[5] C. Tao, "Astrophysical Constraints on Dark Matter," arXiv: 1110.0298.

[6] H. de Vega and N. Sanchez, "Highlights and Conclusions of the Chalonge Workshop 2011," arXiv:1109.3187 [astro-ph.CO].

[7] A. Dolgov, "Cosmological Implications of Neutrinos," Surveys High Energy Physics, Vol. 17, No. 1-4, 2002, pp. 91-114. doi:10.1080/0124421021000054202

[8] M. Zombeck, "Handbook of Space, Astronomy and Astrophysics," Cambridge University Press, Cambridge, 1990.

[9] S. Chandrasekhar, "Stellar Structure," University of Chicago Press, Chicago, 1939.

[10] L. Landau and E. Lifshitz, "Statistical Physics," AddisonWesley Publishing Co., Boston, 1969.

[11] J. Huntley and W. Saslaw, "The Distribution of Stars in Galactic Nuclei: Loaded Polytropes," Astrophysical Journal, Vol. 199, 1975, pp. 328-335. doi:10.1086/153695

[12] R. Viollier, F. Leimgruber and D. Trautmann, "Halos of Heavy Neutrinos around Baryonic Stars," Physics Letters B, Vol. 297, No. 1-2, 1992, pp. 132-137. doi:10.1016/0370-2693(92)91081-J

[13] R. Viollier, D. Trautmann and G. Tupper, "Supermassive Neutrino Stars and Galactic Nuclei," Physics Letters, Vol. 306, No. 1-2, 1993, pp. 79-85.

[14] V. Rubin, W. Kent Ford Jr. and N. Thonnard, "Extended Rotation Curves of High-Luminosity Spiral Galaxies. IVSystematic Dynamical Properties, SA through SC," As- 
trophysical Journal, Vol. 225, No. 1, 1978, pp. L107L111.

[15] R. Cowsik and J. McClelland, "Gravity of Neutrinos of Nonzero Mass in Astrophysics," Astrophysical Journal, Vol. 180, 1973, pp. 7-10. doi:10.1086/151937

[16] R. Cowsik, "The Neutrino Hypothesis for Dark Matter in Dwarf Spheroidals," Journal of Astrophysics and Astronomy, Vol. 7, No. 1, 1986, pp. 1-6. doi:10.1007/BF02715021

[17] T. Nieuwenhuizen, "Do Non-Relativistic Neutrinos Constitute the Dark Matter?" Europhysics Letters, Vol. 86, No. 3, 2009, Article ID: 59001.

[18] P. Langacker, J. Leveille and J. Sheiman, "On the Detection of Cosmological Neutrinos by Coherent Scattering," Physical Review D, Vol. 27, No. 6, 1983, pp. 1228-1242.
doi:10.1103/PhysRevD.27.1228

[19] J. Hamann, S. Hannestad, G. Raffelt and Y. Wong, "Sterile Neutrinos with eV Masses in Cosmology-How Disfavoured Exactly?" Journal of Cosmology and Astroparticle Physics, No. 9, 2011, arXiv:1108.4136.

[20] H. de Vega and N. Sanchez, "Model-Independent Analysis of Dark Matter Points to a Particle Mass at the keV Scale," Monthly Notices of the Royal Astronomical Society, Vol. 404, No. 2, 2010, pp. 885-894. doi:10.1111/j.1365-2966.2010.16319.x

[21] P. Biermann and A. Kusenko, "Relic keV Sterile Neutrinos and Reionization," Physical Review Letters, Vol. 96, No. 9, 2006, Article ID: 091301. doi:10.1103/PhysRevLett.96.091301 